\title{
On Truncatable Primes
}

\author{
By I. O. Angell and H. J. Godwin
}

\begin{abstract}
Truncatable primes are those that yield a sequence of primes when digits are removed always from the left or always from the right. The sizes of the largest truncatable primes in a given number base are estimated by a probabilistic argument and compared with computed values.
\end{abstract}

The number 357686312646216567629137 is a prime, and, if successive digits are removed from the left, a sequence of primes ending $137,37,7$ is obtained. We call such a number a left-truncatable prime and this particular one is the largest in decimal notation. Similarly 73939133 is the largest right-truncatable prime to base 10 : it yields a sequence of primes . . 73, 7 if we truncate from the right. The number 1979339339 has been quoted [1] as the largest right-truncatable prime; but we adhere to the convention that 1 is not a prime number, and so exclude it.

We have computed $L_{a}$, the largest left-truncatable prime with base $a$, for $3 \leqslant a$ $\leqslant 11$, and $R_{a}$, the largest right-truncatable prime with base $a$, for $3 \leqslant a \leqslant 15$. The results are as follows (in decimal form).

\begin{tabular}{lll}
\hline \multicolumn{1}{c}{$R_{a}$} & \multicolumn{1}{c}{$L_{a}$} \\
\hline 3 & 71 & 23 \\
4 & 191 & 4091 \\
5 & 2437 & 7817 \\
6 & 108863 & 4836525320399 \\
7 & 6841 & 817337 \\
8 & 4497359 & 14005650767869 \\
9 & 1355840309 & 1676456897 \\
10 & 73939133 & 357686312646216567629137 \\
11 & 6774006887 & 2276005673 \\
12 & 18704078369 & \\
13 & 38901772669 & \\
14 & 6525460043032393259 & \\
15 & 927920056668659 & \\
\hline
\end{tabular}

To investigate the length of the largest truncatable primes we use the fact that the density of primes in the neighborhood of $n$ is $1 / \log n$. Consider first right-

Received June 2, 1976.

AMS (MOS) subject classifications (1970). Primary 10A25; Secondary 60-01, 60-04.

Key words and phrases. Primes, number bases, probability. 
truncatable primes. Given such a prime $p$, with $r$ digits to base $a$, we look for one with $r+1$ digits by testing which of ap, ap $+1, \ldots, a p+a-1$ are prime. (Actually we tested for pseudoprimality: for the longest pseudoprime found, true primality for it and its sequence of truncations was then checked, using tables or Lehmer's test.) The expected number of primes among these is $a / \log \mu$, where $a p \leqslant \mu \leqslant a p+a-1$, i.e. $a^{r-1}<\mu<a^{r}$. In practice, we should consider $a p+t$ where $t$ runs through numbers $1, \ldots, a-1$ prime to $a$ : if $a$ has prime divisors $q_{1}, q_{2}, \ldots, q_{n}$, this means that we have $a \Pi_{i=1}^{n}\left(1-1 / q_{i}\right)$ choices; but now the probability of such a choice being prime is

$$
\frac{1}{\log \mu} \cdot \prod_{i=1}^{n}\left(1-\frac{1}{q_{i}}\right)^{-1}
$$

and the same result is obtained.

If there are $\pi(a)$ primes less than $a$, then the expected number of right-truncatable primes with $s$ digits lies between

$$
\pi(a) \prod_{r=1}^{s-1}\left(\frac{a}{r \log a}\right) \text { and } \pi(a) \prod_{r=1}^{s-1}\left(\frac{a}{(r+1) \log a}\right),
$$

i.e. between

$$
\frac{\pi(a) a^{s-1}}{(s-1) !(\log a)^{s-1}} \text { and } \frac{\pi(a) a^{s-1}}{s !(\log a)^{s-1}} .
$$

Using Stirling's approximation to the factorial, these bounds become

$$
\frac{s \pi(a) \log a}{\sqrt{2 \pi s} a}\left(\frac{a e}{s \log a}\right)^{s} \text { and } \frac{\pi(a) \log a}{\sqrt{2 \pi s} \cdot a}\left(\frac{a e}{s \log a}\right)^{s} \text {. }
$$

These expressions are dominated by $(a e /(s \log a))^{s}$, for growth if $s<a e / \log a$ and for decrease if $s>a e / \log a$. Hence a rough estimate of the maximum $s$ is $a e / \log a$. For small values of $s$ we can obtain a better estimate by replacing $\log \mu$, where $a^{s-1}<$ $\mu<a^{s}$, by the average of $\log x$ over $\left(a^{s-1}, a^{s}\right)$, i.e. by $((s a-s+1) \log a) /(a-1)-1$ : the estimate of the largest $s$ is then the largest value for which the expected number of primes is not less than a half.

For left-truncatable primes, $p$, of length $s$, is followed by primes among $a^{s}+p$, $2 \cdot a^{s}+p, \ldots,(a-1) a^{s}+p$. We now have $(a-1)$ choices; but all the numbers are prime to $a$, and so the expected number of primes is

$$
\frac{(a-1)}{\log \mu} \prod_{i=1}^{n}\left(1-\frac{1}{q_{i}}\right)^{-1}
$$

The crude estimate of $s$ is

$$
\frac{a e}{\log a} \prod_{1}^{n}\left(1-\frac{1}{q_{i}}\right)^{-1}
$$

and a better estimate can be obtained in a similar way to that used for right-truncatable primes, except that now $q_{1}, \ldots, q_{n}$, give rise to no primes of length greater than one. 
It will be noted that we have excluded 0 as a leading digit. If we do not do this, the length of the longest left-truncatable prime becomes indeterminate since, for example, there are, with probability one, infinitely many primes of the form $10^{k}+3$.

The comparison between observed lengths and lengths given by the better estimates are as follows:

\begin{tabular}{|c|c|c|c|c|}
\hline \multirow[t]{3}{*}{$a$} & \multicolumn{4}{|c|}{ Length (in base $a$ ) of } \\
\hline & \multicolumn{2}{|c|}{$R_{a}$} & \multicolumn{2}{|c|}{$L_{a}$} \\
\hline & observed & $\begin{array}{l}\text { better } \\
\text { estimate }\end{array}$ & observed & $\begin{array}{l}\text { better } \\
\text { estimate }\end{array}$ \\
\hline 3 & 4 & 5 & 3 & 5 \\
\hline 4 & 4 & 6 & 6 & 9 \\
\hline 5 & 5 & 7 & 6 & 7 \\
\hline 6 & 7 & 8 & 17 & 19 \\
\hline 7 & 5 & 8 & 7 & 8 \\
\hline 8 & 8 & 9 & 15 & 16 \\
\hline 9 & 10 & 10 & 10 & 13 \\
\hline 10 & 8 & 10 & 24 & 23 \\
\hline 11 & 10 & 11 & 9 & 11 \\
\hline 12 & 10 & 12 & & \\
\hline 13 & 10 & 12 & & \\
\hline 14 & 17 & 13 & & \\
\hline 15 & 13 & 14 & & \\
\hline
\end{tabular}

Department of Statistics and Computer Science Royal Holloway College Egham Hill, Egham

Surrey, TW20 0EX, England

1. H. J. CLARK, Letter in Computer Weekly, No. 360, September 27, 1973, p. 26. 